\title{
Equilíbrio estático da criança com baixa visão por meio de parâmetros estabilométricos
}

\author{
Static balance of low vision children using stabilometric parameters
}

\author{
Márcio Rodrigues de Matos $^{[\mathrm{a}]}$, Carla Paes Gomes de Matos ${ }^{[\mathrm{b}]}$, Claudia Santos Oliveira ${ }^{[\mathrm{c}]}$
}

[a] Mestre em Engenharia Biomédica pela Universidade do Vale do Paraíba (UNIVAP), professor da Faculdade de Pindamonhangaba (FAPI), Pindamonhangaba, SP - Brasil, e-mail: marciomatos@fapi.br

[b] Mestre em Saúde da Criança e do Adolescente (Unicamp), professora do Programa de Formação em Odontologia para Pessoas com Deficiência (FOSJC-UNESP), São José dos Campos, SP - Brasil, e-mail: carlapaesgomes@hotmail.com

[c] Doutora em Ciências da Saúde pela Universidade de Brasília (UnB), professora do Programa de Mestrado em Ciências da Reabilitação Universidade Nove de Julho (UNINOVE), São Paulo, SP - Brasil, e-mail: csantos@uninove.br

\section{Resumo}

Introdução: A visão é considerada um dos mais importantes meios de captação das informações do ambiente, sendo fundamental para o processo de desenvolvimento da criança. Objetivos: Cientes da participação da visão na manutenção do equilíbrio e no desenvolvimento biopsicossocial do ser humano e considerando os fatores decorrentes da perda ou limitação visual, esta pesquisa objetivou analisar o equilíbrio em postura ortostática de crianças com baixa visão, de faixa etária de 8 a 11 anos, de ambos os sexos (grupo experimental), comparando com o equilíbrio de crianças normais de ambos os sexos e mesma faixa etária (grupo controle), por meio de parâmetros estabilométricos. Materiais e métodos: Para a obtenção das oscilações posturais (deslocamento radial e velocidade de deslocamento) foi utilizada uma plataforma estabilométrica. Resultados: Considerando-se os índices (média e desvio padrão) de deslocamento radial apresentados pelos grupos experimental e controle, observou-se que não houve diferença significante na condição olhos fechados ( $p>0,05)$. Já na condição olhos abertos, houve maior deslocamento radial do grupo experimental em relação ao grupo controle $(\mathrm{p}<0,05)$. Analisando-se os índices (média e desvio padrão) de velocidade de deslocamento entre os grupos controle e experimental, observou-se que em ambas as condições (olhos abertos e olhos fechados) o grupo controle apresentou maior velocidade de deslocamento quando comparado ao grupo experimental $(\mathrm{p}<0,01)$. Conclusão: A partir dos resultados apresentados, concluímos que a baixa visão parece influenciar negativamente a estabilidade em postura ortostática, bem como a velocidade de ajuste postural, prejudicando o equilíbrio corporal.

Palavras-chave: Criança. Baixa visão. Deficiência visual. Equilíbrio. Estabilometria. 


\begin{abstract}
Introduction: Vision is considered one of the most important means of capturing the information from the environment. It is central to the process of child development. Objective: Aware of the contribution of vision on balance maintenance and on human biopsychosocial development, and considering the factors resulting from loss or impairment of vision, this research objected to analyze the balance on orthostatic posture of low vision children, from 8 to 11 years old, of both genders (experimental group) comparing with normal children balance of both genders and same age (control group) using stabilometric parameters. Materials and methods: A stabilometric plataform was used to measure the postural oscillations (radial displacement and displacement speed). Results: Considering the experimental and control groups radial displacement rates (media and standard diversion), it could be observed that there was no significant difference on the closed eyes condition $(p>0,05)$. On the opened eyes condition, there was a bigger radial displacement of the experimental group comparing to the control group $(p<0,05)$. Analysing the displacement speed rates of the control and experimental groups, it could be observed that in both conditions (closed and opened eyes) the control group presented the higher displacement speed when compared to the experimental one $(p<0,01)$. Conclusion: After the presented results, we concluded that low vision might have a negative influence on the orthostatic posture stability as well as on the postural adjustment speed, damaging the body balance.
\end{abstract}

Keywords: Children. Low vision. Visual impairment. Balance. Stabilometry.

\title{
Introdução
}

A deficiência visual (DV) é caracterizada pela cegueira ou baixa visão. Em um levantamento de pesquisas realizadas pela Organização Mundial da Saúde (OMS), no ano de 2002, considerando a Classificação Internacional das Doenças em sua décima revisão (CID-10), estima-se que o número de pessoas com deficiência visual está acima de 161 milhões, das quais aproximadamente 37 milhões são cegos. E ainda, considerando-se um grupo de países (Brasil, Paraguai e Barbados), estimou-se uma prevalência de 0,3\% de pessoas cegas e $1,7 \%$ de pessoas com baixa visão na faixa etária de até 15 anos de idade (1).

No Brasil, estudos de diversos serviços de atendimento oftalmológico à criança referem à toxoplasmose congênita e à catarata congênita (secundária à rubéola) como principais causas de DV na infância (2).

A baixa visão coloca o indivíduo em um tipo de área cinzenta, entre a visão normal e a cegueira completa. Esta área engloba vários níveis de desempenho visual, que vão de um extremo ao outro $(3,4)$.

A visão é considerada um dos mais importantes meios de captação das informações do ambiente, sendo fundamental para o processo de desenvolvimento da criança $(2,5-7)$. Desse modo, o desenvolvimento motor e a capacidade de comunicação são prejudicados na criança com deficiência visual, porque gestos e condutas sociais são aprendidos pelo feedback visual (7).

As crianças com baixa visão utilizam sua visão residual para explorar o ambiente e, embora se diferenciem muito nas suas possibilidades visuais, necessitam aprender a utilizar a visão da melhor forma possível, já que o déficit visual pode levar a um prejuízo no desenvolvimento sensório-motor, cognitivo e da linguagem (8).

O desenvolvimento neuromotor dos lactentes com DV encontra-se comprometido em quase todos os casos de diminuição grave da visão. Em geral, as capacidades motoras estáticas, aquelas necessárias para manter posições ou posturas estacionárias, como sentar e permanecer de pé, desenvolvem-se com normalidade. Porém, as capacidades dinâmicas (mover-se de uma posição ou lugar para outro), como rastejar, engatinhar e deambular e uso das mãos, frequentemente se atrasam $(9,10)$.

Em um estudo envolvendo 14 lactentes cegos, foi verificado que o desenvolvimento motor é afetado desde o primeiro mês de vida. Para o autor, a alteração se deve principalmente à calibração que o sistema visual deixa de exercer sobre o sistema proprioceptivo e vestibular (11).

O diagnóstico precoce de doenças que determinam prejuízo visual permite um tratamento efetivo, e quando não é possível tratar a doença ou a lesão é cicatricial, a prescrição de auxílios óticos e um programa de estimulação visual precoce permitem que a criança possa ter uma integração maior com seu meio (12). 
A intervenção precoce poderia auxiliar na compensação da perda da calibração visual sobre os outros sistemas sensoriais (11) e, ainda, amenizar os comprometimentos advindos da perda da visão (13).

Em um estudo investigando o equilíbrio de crianças cegas, utilizando o Exame Neurológico Evolutivo (ENE) de Lefèvre, foi verificado déficit de equilíbrio e coordenação apendicular (14).

Além disso, outros autores também identificaram déficit de equilíbrio na criança cega, utilizando o ENE associado à coleta de dados por meio da plataforma de força Cybex Reactor ${ }^{\circledR}$ (15).

Os sistemas sensoriais relacionados ao equilíbrio corporal incluem a visão, a propriocepção e o sistema vestibular (16).

Vários estudos foram realizados procurando examinar a predominância das informações visuais no controle postural em crianças normais nas condições com e sem visão e, de forma geral, revelaram que o uso da informação visual durante a manutenção da posição em pé é alterado conforme o desenvolvimento se processa (17-24).

De acordo com alguns autores, mudanças desenvolvimentais no controle postural estão relacionadas à melhora no processo de integração das informações provenientes dos sistemas sensoriais. Nesta proposta, o controle postural em crianças é alcançado prioritariamente com base nas informações visuais. Somente por volta dos 7 anos de idade é que ocorre um período de transição, no qual o sistema de controle postural deixa de ser estritamente dependente da visão e passa a integrar as informações provenientes dos demais sistemas sensoriais para o controle da postura, assumindo, então, uma estratégia semelhante à verificada no funcionamento do sistema de controle postural em adultos $(25,26)$.

Por volta do $7^{\circ}$ ano de vida, a informação visual parece produzir efeito significativo na redução das oscilações corporais durante a manutenção da posição em pé (17).

Entretanto, em estudo utilizando um emissor infravermelho (OPTOTRAK $3020^{\circledR}$ ) posicionado no centro de massa, objetivando examinar a oscilação corporal durante a manutenção da posição estática em pé e o papel da informação visual na manutenção desta posição, conclui-se que os resultados sugerem que nenhuma mudança desenvolvimental drástica ocorre ao longo dos 2 aos 6 anos de idade no controle postural necessário para manutenção em pé (27).

Os mesmos autores ainda acrescentam que o uso da informação visual parece não melhorar significativamente a manutenção desta posição, sugerindo que o controle postural em crianças, nesta faixa etária, não depende prioritariamente da visão.

Cientes da participação da visão na manutenção do equilíbrio e no desenvolvimento biopsicossocial do ser humano e considerando os fatores decorrentes da perda ou limitação visual, esta pesquisa objetivou analisar o equilíbrio em postura ortostática de crianças com baixa visão, de ambos os sexos, com idade entre 8 a 11 anos (grupo experimental), comparando com o equilíbrio de crianças normais de ambos os sexos e mesma faixa etária (grupo controle), por meio de parâmetros estabilométricos.

\section{Materiais e métodos}

A pesquisa realizada tratou-se de um estudo descritivo transversal controlado. Para este estudo, compondo o grupo experimental, foram selecionadas oito crianças com baixa visão, de faixa etária de 8 a 11 anos, de ambos os sexos (cinco meninos e três meninas), atendidas no Centro de Prevenção e Reabilitação de Deficiência da Visão (Provisão), situado na cidade de São José dos Campos, SP, também matriculadas em escolas municipais da mesma cidade.

Para compor o grupo controle, selecionaram-se oito crianças normais, de mesma faixa etária, de ambos os sexos (cinco meninos e três meninas), matriculadas em uma escola municipal de ensino fundamental do mesmo município.

Foram considerados fatores de inclusão: autorização da diretoria da instituição de reabilitação em deficiência visual e da diretoria da escola municipal, para realização da pesquisa; crianças com diagnóstico oftalmológico de baixa visão (para o grupo experimental); autorização por parte dos pais ou responsáveis por meio do Termo de Consentimento Livre e Esclarecido. O critério de exclusão foi: criança que tenha indicação 
de correção oftalmológica por lentes refracionais não estar fazendo o uso delas no momento da coleta de dados na plataforma de força.

A pesquisa foi realizada no Laboratório de Análise de Marcha e Equilíbrio, do Instituto de Pesquisa e Desenvolvimento (IP\&D) da Universidade do Vale do Paraíba (Univap), São José dos Campos, SP.

Este estudo envolvendo seres humanos foi aprovado pelo Comitê de Ética e Pesquisa da Univap, protocolo n. 1.044/2005, sendo certificado de estar conforme as diretrizes e normas regulamentadoras do Conselho Nacional de Saúde.

Sendo o pesquisador membro da equipe de reabilitação da instituição em que as crianças com baixa visão eram atendidas, ele teve acesso aos seus prontuários, identificando dados referentes às avaliações oftalmológicas e ortópticas.

Para mensuração do equilíbrio estático utilizou-se a estabilometria, definida como um método de avaliação do equilíbrio na postura ortostática enquanto o indivíduo permanece sobre uma plataforma de força (28).

A plataforma de força empregada pertence ao Sistema de Análise FootWork ${ }^{\circledR}$, com 2.704 captadores capacitivos de 7,62 x 7,62 mm. Este equipamento é composto por um conversor A/D de 16 bits e uma frequência de amostragem de $250 \operatorname{Hertz}(\mathrm{Hz})$.

Os parâmetros mensurados foram os deslocamentos nos eixos ântero-posterior (y) e deslocamentos nos eixos médio-lateral (x), e ainda a velocidade de deslocamento do centro de pressão (CP). A medida do CP durante a postura em pé tem sido por décadas a principal ferramenta biomecânica para o entendimento do equilíbrio corporal (28-30).

O peso corporal das crianças foi medido em uma balança mecânica Filizola ${ }^{\circledR}$, com precisão de $0,1 \mathrm{~kg}$. As crianças permaneciam descalças, posicionadas em pé, no centro da plataforma da balança e vestindo roupa leve. A estatura foi medida em um estadiômetro fixo, de madeira, com precisão de $0,1 \mathrm{~cm}$. As crianças permaneciam na posição ortostática, descalços, voltados de costas para a superfície vertical do aparelho e com a cabeça posicionada no plano de Frankfurt; os membros superiores relaxados ao lado do tronco, com a palma das mãos voltadas para a coxa; os calcanhares deviam permanecer unidos, tocando a parte vertical do estadiômetro, e a borda medial afastada. A parte móvel do estadiômetro era trazida até tocar o vértex, com compressão do cabelo.

Para a avaliação do equilíbrio de todas as crianças deste estudo, primeiramente foram descritos os procedimentos a serem realizados, bem como a apresentação do equipamento (plataforma estabilométrica). Permitiu-se reconhecimento tátil prévio da plataforma de força pelas crianças.

As crianças foram posicionadas em postura ereta irrestrita, alvo visual localizado aproximadamente a um metro de distância e posicionado na altura da região glabelar de cada uma, com os pés descalços e relaxados sobre a plataforma, calcanhares posicionados à frente da demarcação na plataforma.

Antes do início da coleta dos dados, foi certificado que as crianças estavam identificando o alvo visual (de cor contrastante) e, uma vez posicionadas, em ortostatismo sobre a plataforma, foram orientadas a manter o olhar no alvo visual e a permanecer relaxadas com os braços ao longo do corpo.

Foram realizadas duas aquisições, uma de olhos abertos $(\mathrm{OA})$ e, em seguida, uma com os olhos fechados (OF). O tempo de coleta do sinal de cada aquisição foi de 20 segundos, intercaladas por um período padrão de descanso de um minuto.

A estatística descritiva foi realizada com a utilização do software Excel $97^{\circledR}$, por meio do qual foram calculados as médias e o desvio padrão dos dados antropométricos de ambos os grupos.

O software Microcal Origin ${ }^{\circledR} 6.0$ foi utilizado na estatística inferencial, para realizar a análise comparativa por meio do teste T-Student Independente, com índice de significância de p $<0,05$ para os dados estabilométricos obtidos e na montagem dos gráficos com os resultados alcançados, comparandose as condições estudadas, olhos abertos e olhos fechados, entre as crianças do grupo experimental e do grupo controle.

Os dados referentes à oscilação postural nos sentidos ântero-posterior (x) e médio-lateral (y) do baricentro corporal foram analisados por meio do software utilizando a linguagem Visual Basic, com o auxílio de um analista de sistemas, para exportar os dados e fazer os cálculos para a obtenção das seguintes variáveis: 


\section{Velocidade de deslocamento $(\mathrm{P})$}

Define-se a velocidade de deslocamento $(\mathrm{P})$ como a distância média percorrida por segundo durante o período de tempo da amostra, sendo representada pela fórmula seguinte:

$$
P=\frac{f}{(N-1)} \sum_{i=1}^{N-1} \sqrt{\left\{\left(x_{i+1}-x_{i}\right)^{2}+\left(Z_{i+1}-Z_{i}\right)^{2}\right\}}
$$

Portanto, quanto maior a velocidade de deslocamento, maior a velocidade com que a criança buscou manter-se em equilíbrio.

\section{Deslocamento radial $(\mathbf{R d})$}

A fórmula para calcular os valores do deslocamento radial $(\mathrm{Rd})$ está representada a seguir:

$$
R d=\frac{1}{N} \sum_{i=1}^{N} \sqrt{\left\{\left(x_{i}-x_{c}\right)^{2}+\left(z_{i}-z_{c}\right)^{2}\right\}}
$$

Onde: $\quad x_{c}=\frac{1}{N} \sum_{i=1}^{N}\left(x_{i}\right) \quad z_{c}=\frac{1}{N} \sum_{i=1}^{N}\left(z_{i}\right)$

Em se tratando do deslocamento radial, quanto maior seu índice, menor estabilidade postural a criança apresentou.

\section{Resultados}

As crianças do grupo experimental apresentaram idade de 8 a 11 anos (9,6 $\pm 1,06)$, altura de 1,30 $\mathrm{m}$ a $1,51 \mathrm{~m}(1,37, \pm 7,50)$ e peso entre $22 \mathrm{~kg}$ e $33 \mathrm{~kg}(27,62 \pm 4,03)$. As crianças do grupo controle também apresentaram idade de 8 a 11 anos (9,6 \pm 1,06), altura de 1,29 m a 1,55 m (1,42 \pm 9,56) e peso de $25 \mathrm{~kg}$ a $44 \mathrm{~kg}(33 \pm 7,40)$.

Considerando-se os índices (média e desvio padrão) de deslocamento radial apresentados pelos grupos experimental e controle, observou-se que não houve diferença estatística significante entre eles na condição olhos fechados ( $\mathrm{p}>0,05)$, sendo o valor para baricentro do corpo $\mathrm{p}=0,37$, conforme Gráfico 1.

Já na condição olhos abertos, houve diferença estatística significante, havendo maior deslocamento radial do grupo experimental em relação ao grupo controle $(\mathrm{p}<0,05)$, sendo o valor para baricentro do corpo $\mathrm{p}=0,04$, conforme Gráfico 2.

Analisando-se os índices (média e desvio padrão) de velocidade de deslocamento (baricentro do corpo) entre os grupos controle e experimental, observou-se que em ambas as condições (olhos abertos e olhos fechados) o grupo controle apresentou maior velocidade de deslocamento quando comparado ao grupo experimental, com diferença estatística significante $(\mathrm{p}<0,05)$.

O valor encontrado para a condição olhos abertos no baricentro do corpo foi de $p=9,78 \mathrm{E}-4$. Já para a condição olhos fechados o valor encontrado para o baricentro do corpo foi $\mathrm{p}=2,05 \mathrm{E}-4$, conforme os Gráficos 3 e 4. 


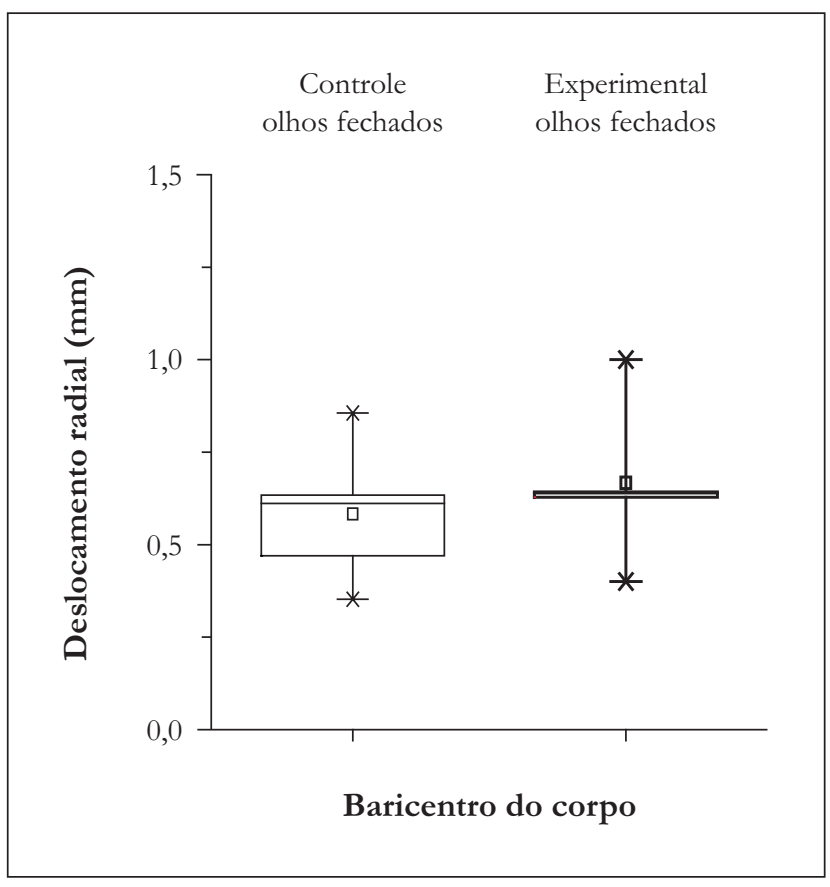

Gráfico 1 - Valores médios e desvio padrão de deslocamento radial (baricentro do corpo) dos grupos controle e experimental na condição olhos fechados

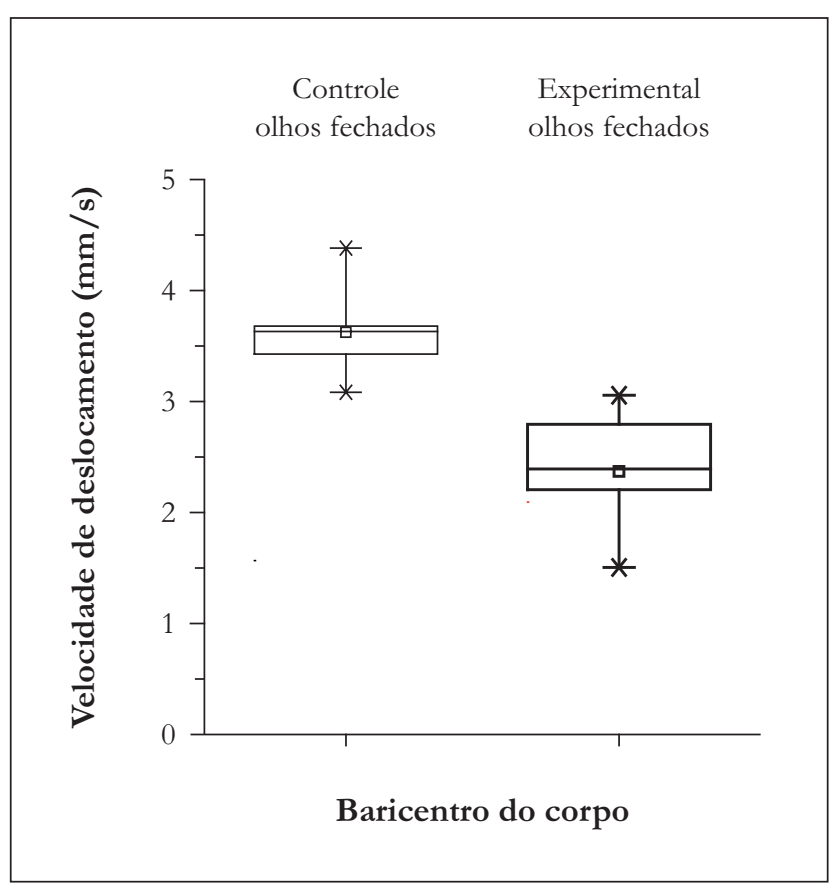

Gráfico 3 - Valores médios e desvio padrão de velocidade de deslocamento (baricentro do corpo) dos grupos controle e experimental na condição olhos fechados

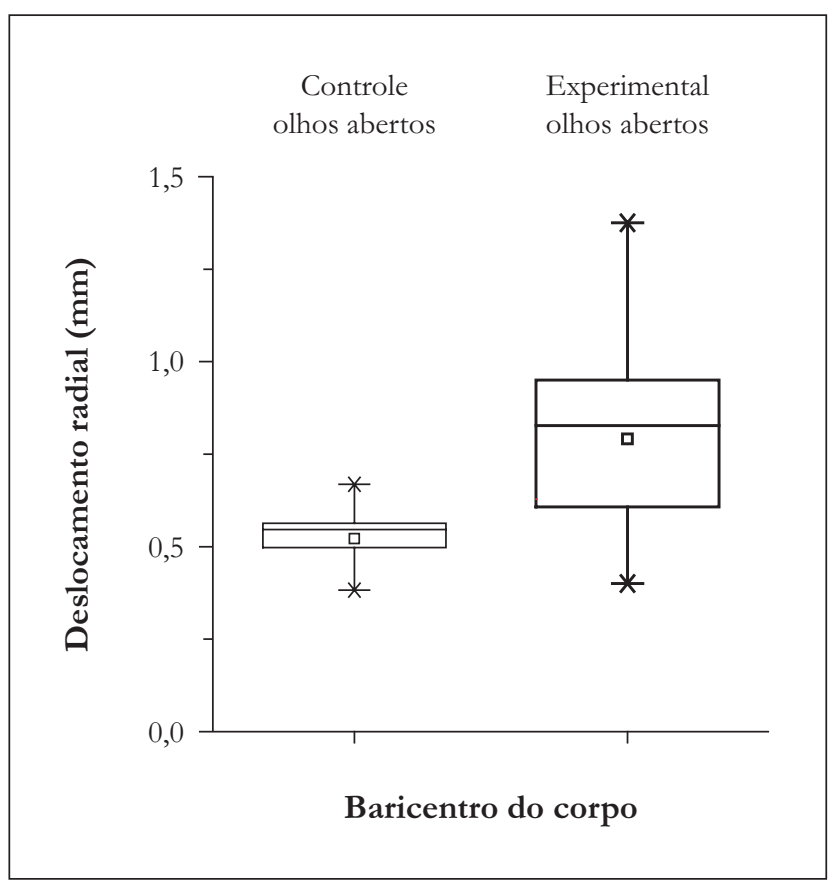

Gráfico 2 - Valores médios e desvio padrão de deslocamento radial (baricentro do corpo) dos grupos controle e experimental na condição olhos abertos

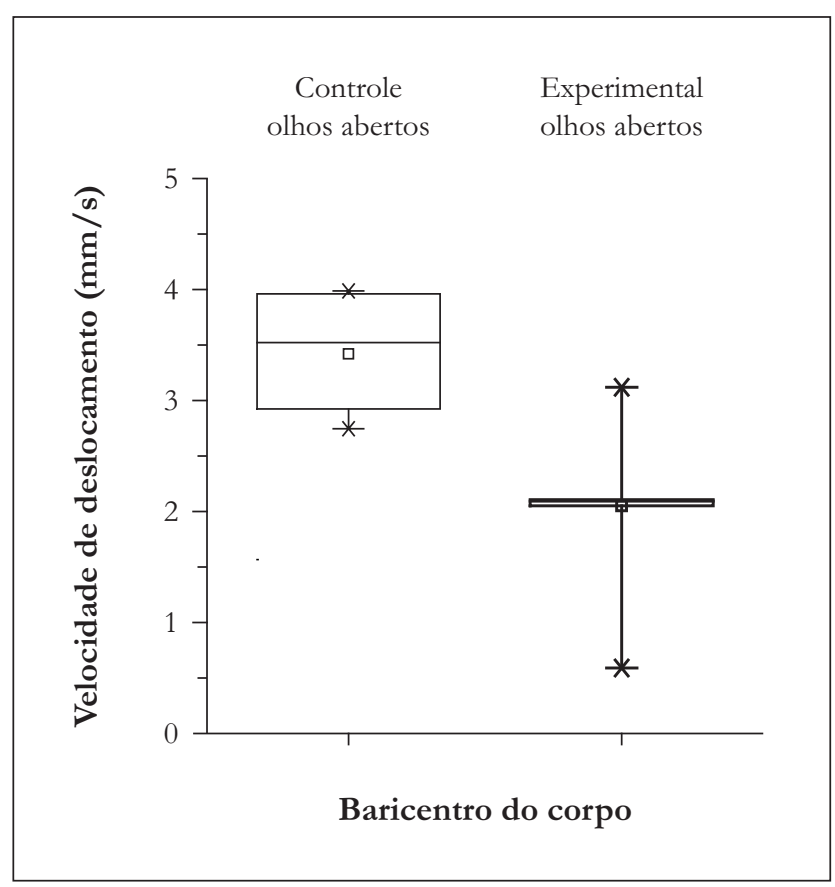

Gráfico 4 - Valores médios e desvio padrão de velocidade de deslocamento (baricentro do corpo) dos grupos controle e experimental na condição olhos abertos 


\section{Discussão}

Os índices de deslocamento radial (baricentro do corpo), quando comparados os grupos controle e experimental na condição olhos fechados, não apresentaram diferença estatística significante.

Entretanto, na condição olhos abertos, para esse mesmo parâmetro, puderam ser evidenciados maiores índices de deslocamento radial, com diferença estatística significante, das crianças do grupo experimental em relação aos apresentados pelas do grupo controle, indicando prejuízo da estabilidade postural em ortostatismo das crianças com baixa visão.

Por volta do $7^{\circ}$ ano de vida, a visão parece produzir efeito significativo na redução das oscilações corporais durante a manutenção da posição em pé (17), o que fala em favor dos resultados obtidos no presente estudo, visto que as crianças apresentavam faixa etária de 8 a 11 anos, e o dano da acuidade visual (no caso das crianças com baixa visão) pode estar influenciando, neste caso negativamente, o equilíbrio estático delas quando comparadas às crianças normais.

Se considerarmos as pesquisas que afirmam que somente por volta dos 7 anos de idade é que ocorre um período de transição em que o sistema de controle postural deixa de ser estritamente dependente da visão e passa a integrar as informações provenientes dos demais sistemas sensoriais para o controle da postura (25, 26), podemos entender que, sendo a presente pesquisa conduzida com crianças com mais de 7 anos de idade, a visão ainda pode estar interferindo na manutenção da postura ortostática.

Com relação ao parâmetro velocidade de deslocamento, o grupo controle apresentou maiores índices para o baricentro do corpo do que o grupo experimental em ambas as condições, olhos fechados e olhos abertos, ou seja, as crianças normais apresentaram maior velocidade para promover ajustes posturais do que as crianças com baixa visão, mesmo na condição olhos fechados, em que a situação visual de ambos os grupos se equipararam.

Estes dados permitem considerar que o prejuízo do equilíbrio estático apresentado pelas crianças com baixa visão pode ser decorrente do déficit de acuidade visual por si só, mas também ser consequente dos atrasos no desenvolvimento motor (7) e sensório-motor (8) secundários a este déficit.

No entanto há autores que afirmam que em lactentes deficientes visuais as habilidades motoras estáticas desenvolvem-se com normalidade $(9,10)$, porém a diferença relativa à faixa etária entre a presente pesquisa e o referido estudo e o fato de que o uso da informação visual durante a manutenção da posição em pé é alterado desenvolvimentalmente (17-21) podem justificar a disparidade entre os resultados obtidos.

Acredita-se que a alteração do desenvolvimento motor da criança cega se deve principalmente à calibração que o sistema visual deixa de exercer sobre o sistema proprioceptivo e vestibular (11), o que também poderia estar presente nas crianças com baixa visão deste estudo, justificando os déficits de estabilidade e ajuste postural apresentados.

Pesquisas anteriores já mencionavam prejuízo do equilíbrio estático em crianças cegas $(14,15)$, sendo assim o presente estudo indica que nos casos de baixa visão investigados a mesma condição também se faz presente.

Devemos considerar também que, em se tratando de baixa visão, há vários níveis de desempenho visual, que vão de um extremo ao outro $(3,4)$, e que, portanto, não há possibilidade de se estender os resultados apresentados pelas crianças do grupo experimental desta pesquisa a uma diversidade de casos de baixa visão.

Apesar de não terem sido investigados com maior profundidade os dados referentes ao atendimento reabilitacional de cada criança com deficiência visual, sendo a plasticidade neuronal um fato, a intervenção precoce poderia auxiliar na compensação da perda da ação visual sobre os outros sistemas sensoriais, podendo a intervenção terapêutica amenizar os comprometimentos advindos da perda da visão $(11,13)$.

\section{Considerações finais}

Concluímos, por meio desta pesquisa, que a baixa visão apresentada pelas crianças (de faixa etária de 8 a 11 anos) deste estudo parece influenciar negativamente o deslocamento radial, bem como a velocidade de deslocamento, o que pode prejudicar seu equilíbrio em postura ortostática quando comparados a crianças 
normais de mesma faixa etária, considerando-se as informações adquiridas por meio dos parâmetros da plataforma estabilométrica.

Contudo, merece ser melhor investigado o contexto social em que as crianças estão inseridas, considerando-se aspectos de desenvolvimento neuropsicomotor e reabilitacional, que também podem interferir na manutenção do equilíbrio delas.

\section{Agradecimentos}

À Coordenação de Aperfeiçoamento e Pesquisa no Nível Superior (CAPES), pelo suporte financeiro. Ao Centro de Prevenção e Reabilitação de Deficiência da Visão - PROVISÃO.

À Escola Municipal de Ensino Fundamental "Profa Elza Regina F. Bevilacqua".

À Escola de Terapia Manual e Postural.

\section{Referências}

1. Resnikoff S, Pascolini D, Etya‘Ale D, Kocur I, Pararajasegaram R, Porkharel GP, et al. Global data on visual impairment in the year 2002. Bull World Health Organ. 2004;82(11):844-51.

2. Haddad MAO, Sampaio MW, Kara-José N. Baixa visão na infância: manual básico para oftalmologistas. São Paulo: Laramara; 2001.

3. Colenbrander A, Fletcher DC. Basic concepts and terms for low vision rehabilitation. Am J Occup Ther. 1995;49(9):865-9.

4. Warren M. Providing low vision rehabilitation services with occupational therapy and ophthalmology: a program description. Am J Occup Ther. 1995;49(9):877-83.

5. Helders PJM. Early motor signs of blindness or very low vision in very young children. Early Intervention. 1986; 359-65.

6. Áries MHR, Zeferino AMB, Barros Filho AA. Surdocegueira: importância da estimulação precoce. Temas sobre Desenvolvimento. 2004;77:12-9.

7. Graziano RM, Leone CR. Problemas oftalmológicos mais freqüentes e desenvolvimento visual do pré-termo extremo. J Pediatr. 2005;81(Suppl1);S95-S100.

8. Brasil. Ministério da Educação. Estratégias e orientações pedagógicas para a educação de crianças com necessidades educacionais especiais: dificuldade de comunicação e sinalização (deficiência visual). Brasília: MEC / SEESS; 2002.

9. Hyvärinen L. La vision normal y anormal en los niños. Madrid: Oraginización Nacional de Ciegos Españoles; 1988.

10. Pogrund R, Fazzi DL, Lampert JS. Early focus: working with young blind and visually impaired children and their families. New York: Americam Foundation for the Blin; 1994.

11. Prechtl H, Cioni G, Einspieler C, Bos AF, Ferrari F. Role of vision on early motor development: lessons for the blind. Dev Med Child Neurol. 2001;43(3):198-201.

12. Graziano RM. Exame oftalmológico do recém-nascido no berçário: uma rotina necessária.J Pediatr. 2002;78(3):187-8.

13. Levtzion-Korach O, Tennenbaum A, Schnitzer R, Ornoy A. Early motor development of blind children. J Paediatr Child Health. 2000;36(3):226-9.

14. Navarro AS, Fukujima MM, Fontes SV, Matas SLA, Prado GF. Coordenação motora e equilíbrio não são totalmente desenvolvidos em crianças cegas com 07 anos de idade. Arq Neuropsiquiatric. 2004;62(3-A):654-57. 
15. Gomes CP, Bueno RO, Gagliardo HGRG. Estudo do equilíbrio estático da criança deficiente visual. Temas sobre Desenvolvimento. 2004;77:43-53.

16. Buchanan JJ, Horak FB. Voluntary control of postural equilibrium patterns. Behav Brain Res. 2003;143(2):121-40.

17. Zernicke RF, Gregor RJ, Cratty BJ. Balance and visual proprioception in children. J Hum Movement Stud. 1982;8:1-13.

18. Riach CL, Hayes KC. Maturation of postural control in young children. Dev Med Child Neurol. 1987;29(5):650-8.

19. Riach CL, Starkes JL. Visual fixation and postural sway in children. J Mot Behav. 1989;21(3):265-76.

20. Ashmead DH, McCarty ME. Postural sway of human infants while standing in light and dark. Child Dev. 1991;62(6):1276-87.

21. Portfors-Yeomans C, Riach CL. Frequency characteristics of postural control of children with and without visual impairment. Dev Med Child Neurol. 1995;37(5):456-63.

22. Lee DN, Lishman JR. Visual proprioceptive control of stance. J Hum Mov Stud. 1975;1:87-95.

23. Freitas Júnior PB, Barela JA. Postural control as a function of self- and object-motion perception. Neurosci Lett. 2004;369(1):64-8.

24. Polastri PF, Barela JA. Perception-action coupling in infants with Down syndrome: effects of experience and practice. Adapt Phys Activ Q. 2005;22(1):39-58.

25. Shumway-Cook A, Woollacott MH. The growth of stability: postural control from a developmental perspective. J Mot Behav. 1985;17(2):131-47.

26. Woollacott M, Debû B, Mowatt M. Neuromuscular control of posture in the infant and child: is vision dominant? J Mot Behav. 1987;19(2):167-86.

27. Barela JÁ, Polastri PF, Godoi D. Controle postural em crianças: oscilação corporal e freqüência de oscilação. Rev Paul Educ Fís. 2000;14(1):68-77.

28. Oliveira LF, Simpson DM, Nadal J. Calculation of area of stabilometric signals using principal component analysis. Physiol Meas. 1996;17(4):305-12.

29. Celso CF, Muniz RA, Oliveira LF, Imbiriba LA, Garcia MAC, Magalhães J. Limites de estabilidade ântero-posterior de adultos normais. Anais do IX Congresso Brasileiro de Biomecânica; 2001; Gramado, Brasil.

30. Wieczorek SA, Duarte M, Zatsiorski VM. Manutenção do equilíbrio na postura ortostática em diferentes posições do corpo. Anais do IX Congresso Brasileiro de Biomecânica; 2001; Gramado, Brasil. São Paulo, USP; 2001.

Recebido: 19/11/2008

Received: $11 / 19 / 2008$

Aprovado: 28/03/2010

Approved: 03/28/2010

Revisado: 23/06/2010

Reviewed: 06/23/2010 